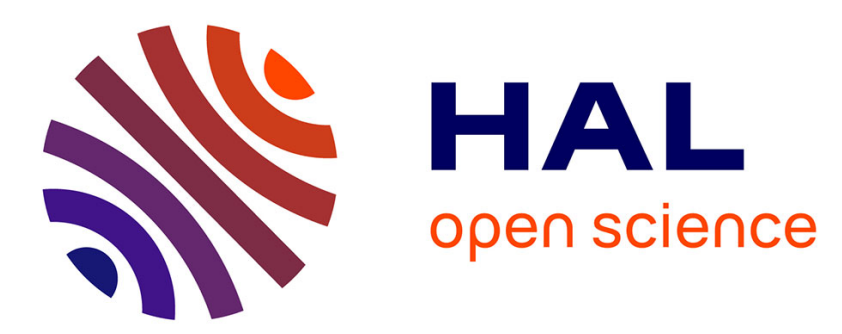

\title{
Magnetic Field Investigations of Small Sputtered Step-Edge Junctions
}

M. Vaupel, G. Ockenfuss, R. Wördenweber

\section{To cite this version:}

M. Vaupel, G. Ockenfuss, R. Wördenweber. Magnetic Field Investigations of Small Sputtered Step-Edge Junctions. Journal de Physique IV Proceedings, 1996, 06 (C3), pp.C3-383-C3-389. 10.1051/jp4:1996358 . jpa-00254276

\section{HAL Id: jpa-00254276 https://hal.science/jpa-00254276}

Submitted on 1 Jan 1996

HAL is a multi-disciplinary open access archive for the deposit and dissemination of scientific research documents, whether they are published or not. The documents may come from teaching and research institutions in France or abroad, or from public or private research centers.
L'archive ouverte pluridisciplinaire HAL, est destinée au dépôt et à la diffusion de documents scientifiques de niveau recherche, publiés ou non, émanant des établissements d'enseignement et de recherche français ou étrangers, des laboratoires publics ou privés. 


\title{
Magnetic Field Investigations of Small Sputtered Step-Edge Junctions
}

\author{
M. Vaupel, G. Ockenfuss and R. Wördenweber \\ Institut für Schicht- und Ionentechnik(ISI), Forschungszentrum Jülich GmbH, 52425 Jülich, Germany
}

\begin{abstract}
YBa}_{2} \mathrm{Cu}_{3} \mathrm{O}_{7}$ step-edge junctions are fabricated on $\mathrm{SrTiO}_{3}$-substrates. The steps of $300 \mathrm{~nm}$ height are milled by argon-ion-beam-etching ([BE). The films are deposited by high-pressure on-axis magnetron sputter technique pattemed to microbridges with widths down to $0.5 \mu \mathrm{m}$ by electron beam lithography and argon ion etching. For ratios of film thickness to $s t e p$ height of $t / h \approx 1 / 2$ the current-voltage characteristics show Shapiro steps under microwave irradiation and RSJ-(resistively shumted junction)-like behavior. The periodic dependence of the critical current upon the magnetic field resembles a Fraunhofer-pattern. The period of the variation $\Delta B_{0}$ follows a $1 / \mathrm{w}^{2}$-dependence in agreement with the theoretical prediction for planar thin Josephson junctions: $\Delta \mathbf{B}_{0}=1.84 \phi_{0} / \mathrm{w}^{2}$. Junctions with widths of $0.7 \mu \mathrm{m}$ possess a large magnetic field stability with $\Delta B_{0} \approx 100 \mathrm{G}$. Small junctions $(\mathrm{w}<1 \mu \mathrm{m})$ exhibit voltage jumps in the Fraunhofer pattern, which are explained by flux penetration of single vortices into the superconducting electrodes.

The developed preparation technique for 'field-stable' Josephson junctions will be used for wafer scaling on 2-inch substrates and for the production of magnetically stable if-SQUIDs.
\end{abstract}

\section{INTRODUCTION}

Josephson junctions are the basic active components in superconducting thin film electronics. Due to the characteristic parameters of the ceramic high-temperature superconductors (HTS), particularly the extremely short coherence length $\xi_{s}$, the development and optimization of new types of Josephson junctions (JJ) became necessary. The step-edge junction (SEJ) [1] possesses the advantage of good transport properties, low $1 / \mathrm{f}$-noise, remarkably simple fabrication and circuit layout flexibility. It consists of two tilt angle grain boundary junctions in series created by depositing a superconducting film across a steep step in the substrate and patterning of a mikrobridge. For the development of practical junctions for applications, the field dependence of the critical current, $I_{c}(B)$, is of major interest. It reflects not only the homogeneity of the junction, but determines the field stability in SQUID magnetometer applications.

For conventional low-temperature superconductor vertical tunnel junctions, the field dependence of the critical current density is essentially the modulus of the Fourier transform of the critical current, which is usually referred to as a Fraunhofer pattern similar to the diffraction pattern of a single slit. In case of an ideal vertical junction the period of the field dependence is given by

$$
\Delta \mathrm{B}_{0}=\phi_{0} /(2 \lambda w)
$$


with the width $w$ of the junction and $\lambda=\lambda_{\mathrm{L}}$ ( $\lambda_{\mathrm{L}}$ : London penetration depth) in case of thick superconducting electrodes, and $\lambda=\lambda_{\text {eff }}$ ( $\lambda_{\text {eff: }}$ effective penetration depth) for thin superconducting electrodes [2]. For our thin films, the penetration depth is given by $\lambda_{\text {eff }}=\lambda_{L} \operatorname{coth}\left(t / 2 \lambda_{L}\right) \approx \lambda_{\mathrm{L}}{ }^{2} / t$, where $t$ represents the thickness of the electrodes. The magnetic field behavior of planar junctions differs from the ideal behavior given by Eq. (1), due to flux focusing effects of the superconducting electrodes, which automatically lead to a $1 / w^{2}$-dependence of $\Delta B_{0}$. Based upon flux concentration in the junction barrier due to the Meissner effect, for thick planar devices

$$
\Delta \mathbf{B}_{0}=\Phi_{0} t /\left(1.2 w^{2} 2 \lambda_{L}\right)
$$

is predicted [3]. For thin films, flux focusing effects due to vortex trapping in the electrodes are expected to cause a periodicity $[3,4]$ :

$$
\Delta \mathbf{B}_{0}=1.84 \Phi_{0} /\left(\mathrm{w}^{2}\right)
$$

However, Eqs. (2) and (3) are only exact in the extreme thin and thick limits, respectively. Moreover, in the derivations the crossection of the superconducting electrodes normal to the film surface has been approximated by an ellipsoid with an aspect ratio given by the film thickness to the width of the junction. This approximation is questionable since effects like a singularity of the field at the edge of the superconducting film [5] or a belt of lower field [6] caused by a rectangular geometry are neglected in this approach. Therefore, the width dependence of the period $\Delta \mathbf{B}_{0}$ measured over a broad range of $w$ is interesting and important for the understanding of the flux distribution in small planar Josephson junctions. Especially thin SEJ's are expected to show pronounced effects due to flux trapping and penetration.

In this paper, we present the field dependence of the critical current of small sputtered $\mathrm{YBa}_{2} \mathrm{Cu}_{3} \mathrm{O}_{7}$ (YBCO) SEJ's with widths ranging from $\mathrm{w}=2 \mu \mathrm{m} \approx \lambda_{\mathrm{j}}=\left(\mathrm{h} / 8 \mathrm{e} \pi \mu_{0} \mathrm{~J}_{\mathrm{c}} \lambda\right)^{1 / 2}$ down to $\mathrm{w}=0.5 \mu \mathrm{m}$ « $\lambda_{\mathrm{j}}$. Here $\lambda_{j}$ represents the Josephson penetration depth and $J_{c}$ the critical current density. The magnetic field dependence of $\Delta B_{0}$ measured for these small junctions is compared with the different models, Eqs. (1) to (3). Finally, hysteretic behavior and jumps in the Fraunhofer pattern are analyzed.

\section{JUNCTION PREPARATION}

For a reproducible fabrication of SEJ's from sputtered thin films, the reproducible preparation of steep steps in the substrate and the exact control of the ratio between film thickness and step height, $t / h$, are important. The ratio determines the junction behavior. A step angle $\alpha>45^{\circ}$ is necessary for the formation of two grain boundaries at the upper and lower edge of the step [7]. In $\mathrm{SrTiO}_{3}$-substrates steps with heights of $h \approx 300 \mathrm{~nm}$ and $\alpha \approx 80^{\circ}$ are patterned by optical lithography and $\mathrm{Ar}$ ion milling at $\mathrm{T}=15^{\circ} \mathrm{C}$, ion beam current density $\mathrm{J}=0.5 \mathrm{~mA} / \mathrm{cm}^{2}$, and acceleration voltage $U=500 \mathrm{~V}$ [8]. Epitaxial, c-axis-oriented YBCO films are deposited by high pressure dc-magnetron sputtering at a substrate holder temperature of $850^{\circ} \mathrm{C}$, gas pressure of $90 \mathrm{~Pa}$ of $\mathrm{Ar} / \mathrm{O}_{2}$ (ratio $2: 1$ ), and a dc-power of $4.1 \mathrm{~W} / \mathrm{cm}^{2}$ resulting in a sputter rate of $3.5 \mathrm{~nm} / \mathrm{min}$. The films are free of outgrowths and showed a typical roughness of $5-10 \mathrm{~nm}$ peak-to-peak. The inductively determined critical temperature is typically $T_{c, \text { off }}=87-90 \mathrm{~K}$ and the width of the rocking curve of the (005)-peak $\Delta \omega_{\mathrm{FWHM}} \approx 0.15^{\circ}$.

The microbridges are patterned via standard optical lithography using AZ 5214E photoresist for widths $w \geq 2 \mu \mathrm{m}$ and electron beam lithography utilizing a trilayer of gold-niobium-PMMA for widths down to $0.5 \mu \mathrm{m}$ [9]. The YBCO layer was ion beam etched $\left(\mathrm{J}=0.25 \mathrm{~mA} / \mathrm{cm}^{2}, \mathrm{U}=250 \mathrm{~V}\right)$ at liquid nitrogen temperature. Fig. 1 shows a $0.5 \mu \mathrm{m}$ wide step-edge junction. After patterning the samples are treated by IBE-irradiation at $\mathrm{J}=0.25 \mathrm{~mA} / \mathrm{cm}^{2}$ and $\mathrm{U}=250 \mathrm{~V}$ for removing the gold top-layer and trimming the junctions. 


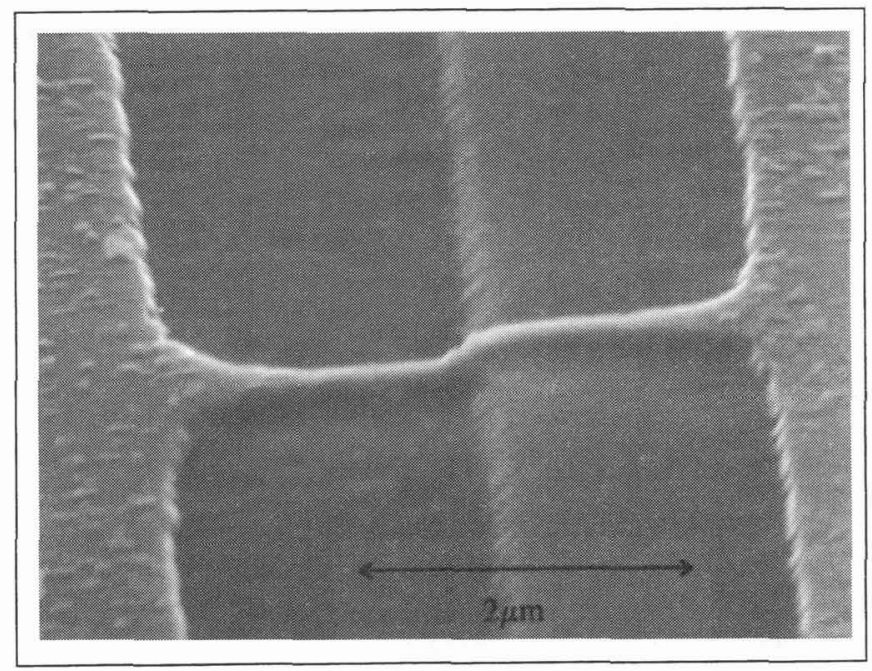

Fig. 1: SEM photo of a $0.5 \mu \mathrm{m}$ wide step-edge junction $(\mathrm{h}=300 \mathrm{~nm}, t=150 \mathrm{~nm})$

\section{RESULTS AND DISCUSSION}

Current-voltage characteristics of steep step-edge junctions depend on the ratio of the film thickness to the step height $t / h$. For $t / h \approx 1 / 2$, our sputtered junctions shows RSJ-like behavior as indicated by Fig. 2a. Shapiro steps appear under microwave irradiation. For smaller ratios, $t / h<1 / 2, T_{c}$ decreases and finally the film is disrupted at the step, whereas for $t / h>1 / 2$ flux flow behavior is observed due to superconductively shunted grain boundaries at the step[10].

Scaling of the $I_{c} R_{n}$ product, where $I_{c}$ is the junction's critical current and $R_{n}$ the normal state resistance, is typical for step-edge junctions: $I_{c} R_{n} \propto J_{c}{ }^{n}$ with $n \approx 0.5-0.8$ [7]. For $t / h \approx 1 / 2$, the scaling of the $I_{c} R_{n}$ values of a set of our SEJ's on a chip according to $I_{c} R_{n} \propto J_{c}{ }^{n}$, with $n \approx 0.76$, (Fig. 2b) is analogous.

The junctions with widths ranging between $0.6 \mu \mathrm{m}$ and $2.5 \mu \mathrm{m}$ possess critical currents of $\mathrm{I}_{\mathrm{c}}(77 \mathrm{~K})$ $=10^{-6}-10^{-5} \mathrm{~A}$ (i.e. $\mathrm{J}_{\mathrm{c}}=10^{3}-10^{4} \mathrm{~A} / \mathrm{cm}^{2}$ ) and $\mathrm{I}_{\mathrm{c}}\left(4.2 \mathrm{~K}\right.$ ) $=10^{-5}-10^{-4} \mathrm{~A}$ (i.e. $\mathrm{J}_{\mathrm{c}}=10^{4}-10^{5} \mathrm{~A} / \mathrm{cm}^{2}$ ). The values of the normal resistance of the as-deposited SEJ range between $R_{n}=1-10 \Omega$. Values up to $\sim 100 \Omega$ are measured after an additional IBE treatment, which provides a powerful possibility for trimming of JJ parameters. Thus, $\mathrm{I}_{\mathrm{c}} \mathrm{R}_{\mathrm{n}}$ products of $0.03-1 \mathrm{mV}$ and $0.2-8 \mathrm{mV}$ are obtained at $77 \mathrm{~K}$ and $4.2 \mathrm{~K}$, respectively.

The magnetic field dependence of the critical current, $I_{c}(B)$, is measured in a magnetic field normal to the film surface. Fig. 3a shows a typical field dependence of the critical current observed in our SEJ's. The figure resembles a Fraunhofer pattern for small junctions, i.e. $w<\lambda_{j}$. The width of the SEJ was $2 \mu \mathrm{m} \approx \lambda_{\mathrm{j}} / 3$. The period $\Delta \mathrm{B}_{\mathrm{o}}$ of the Fraunhofer pattern is derived from these measurements. For 10 $\mu \mathrm{m}$ wide junctions (e.g. data of [3]), the period is roughly equivalent to the magnitude of the earth field, i.e. rotation of a bare junction in unshielded environment would lead to large variations of it's critical current, whereas smaller junctions show a better stability with respect to magnetic fields. Our SEJ's with widths $\mathrm{w}=2 \mu \mathrm{m}$ are 'magnetically stable' with a period $\Delta \mathrm{B}_{0}=8 \mathrm{G}$ and for $0.67 \mu \mathrm{m}$ wide junctions values of $\Delta \mathrm{B}_{0}=65-125 \mathrm{G}$ are measured. 

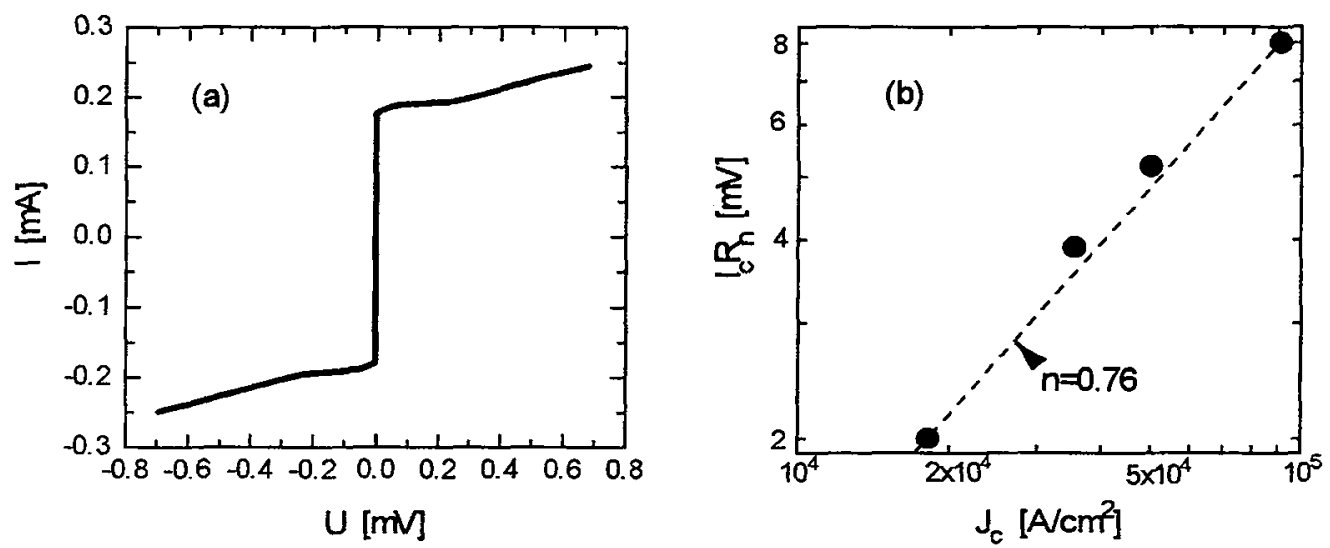

Fig. 2: (a) IV-characteristic of a $0.7 \mu \mathrm{m}$ wide SEJ at $4.2 \mathrm{~K}(t / \mathrm{h}=1 / 2,=150 \mathrm{~nm})$ and (b) $I_{0} R_{\mathrm{n}}(10 \mathrm{~K})$ as a function of the critical current density for a set of $0.7 \mu \mathrm{m}$ wide SES s after IBE-irradiation.

Fig. $3 \mathrm{~b}$ represents a comparison of experimental data and theoretical predictions for the width. dependence of $\Delta \mathbf{B}_{0}$ in a double logarithmic plot. Additional to our experimental values data for biepitaxial junctions [3] are shown. $A$ fit of the experimental data proves the expected behavior $\Delta \mathbf{B}_{0} \approx(1.9 \pm 0.2) \Phi_{0} / \mathbf{w}^{2}$ even down to the sub- $\mu \mathrm{m}$ range. The dependence of $\Delta \mathbf{B}_{0}$ is in excellent agreement with the prediction for thin planar JJ, Eq. (3).
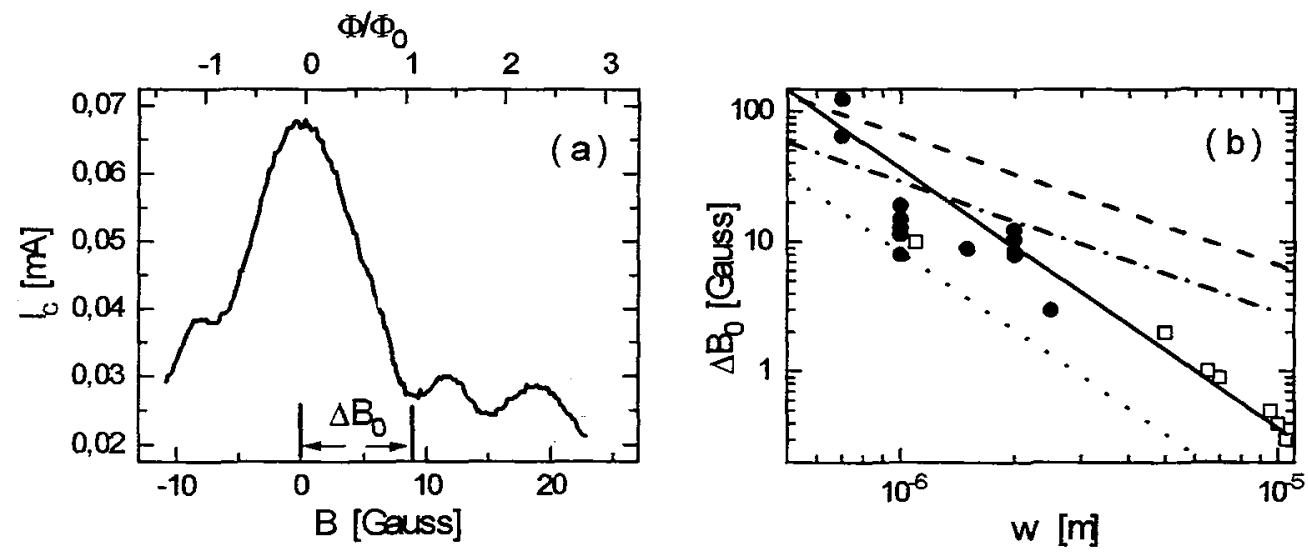

Fig. 3: (a) Field dependence of the critical current of a $2 \mu \mathrm{m}$ wide $\mathrm{SEJ}(\mathrm{t} / \mathrm{h}=1 / 2, \mathrm{t}=120 \mathrm{~nm}$ ) at $40 \mathrm{~K}$. (b) Comparison of the width dependence of the period $\Delta \mathrm{B}_{0}$ obtained from our experimental data (solid circles), experimental data by Rosenthal et al [3] for biepitaxial junctions (open squares) and different theories described be Eq. (1)-(3) with $\lambda_{L}=150 \mathrm{~nm}: \Delta B_{0}=\Phi_{\alpha} /\left(2 \lambda_{L} w\right)$ (dashed line, classical model for thick superconductors), $\Delta B_{0}=\Phi_{0} /\left(2 \lambda_{\text {eff }} w\right)$ (dashed-dotted line, classical model for thin superconductors), $\Delta B_{0}=\Phi_{0} t /\left(1.2 w^{2} 2 \lambda_{L}\right)$ (dotted line, model for planar junctions and thick films) and $\Delta B_{0}=1.84 \Phi_{0} / w^{2}$ (solid line, model for planar junctions and thin films). 
Finally, we concentrate on the effects of flux penetration and flux trapping in our planar SEJ. Shifts or partial suppression of maxima in the Fraunhofer pattem leading to a hysteretic behavior of planar $\mathrm{J} J$ in a magnetic field are commonly observed effects of flux penetration into and flux trapping in the electrodes of HTS thin-film JJ's [11]. These effects become more prominent and illustrative for reduced dimensions of the junctions. In our sub- $\mu \mathrm{m}$-junctions jumps in the $I_{c}$ versus $B$ characteristic occur at external fields at which exactly one flux quantum has entered the effective junction area $A_{\text {eff }} \approx w^{2} / 1.84$. Fig. 4 shows a typical measurement of such jumps at $\pm(85-90) G \approx \pm \Phi_{0} /\left(w^{2} / 1.84\right)$. In order to increase the measuring speed, the voltage drop at the junction, $U_{c}$, has been recorded at constant current as a function of the applied magnetic induction [12].

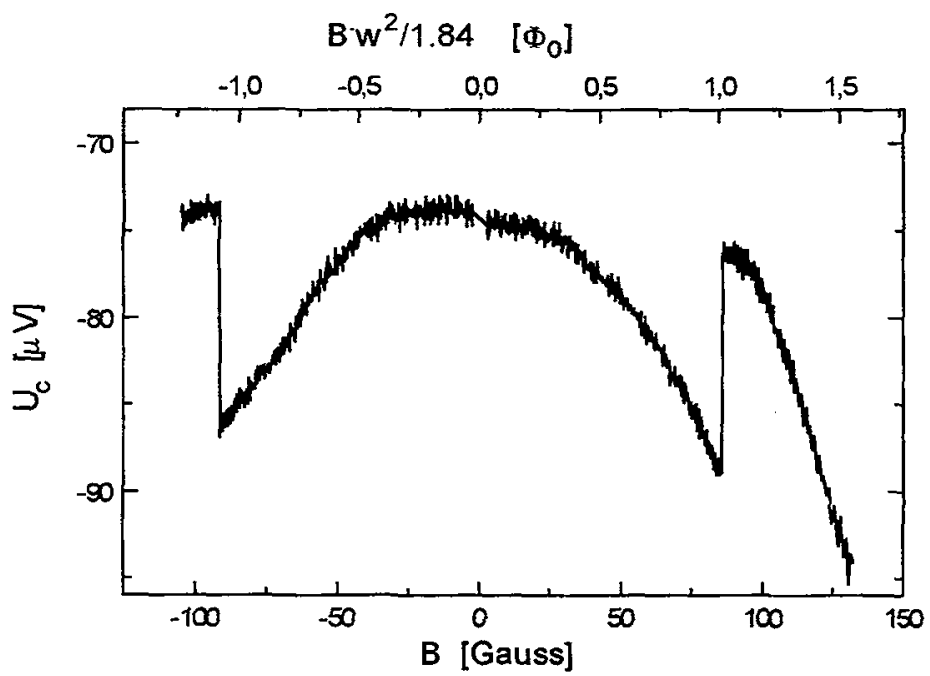

Fig. 4: Jumps of single flux quanta in a $0.7 \mu \mathrm{m}$ wide junction at magnetic induction $B$ equivalent to a single flux quantum, i.e. at $\mathrm{B} \mathrm{w}^{2} / 1.84=\Phi_{0}$

This observation can be explained in terms of a model for homogeneous planar junctions (without weak links in the electrodes) consisting of two electrodes with a rectangular profile normal to the film surface shown in Fig. $5 \mathrm{a}$ and described in the following sections:

(i) Starting from zero-field, magnetic flux $\Phi_{y}$ will enter the effective junction area $A_{\text {eff }}$ along the contact area ( $z \approx 0$ in Fig. 5) when the magnetic induction is first increased. Due to the rectangular shape (in contrast to the often assumed elliptical approach(s. Fig. 5a)) the constant density lines of the screening current in the electrodes (Fig. 5b) lead to a cushion-like flux front (Fig. 5c). This can be obtained from 2D equation of motion for the sheet current in rectangular films with non-linear resistivity in a magnetic field [13]. According to this evaluation a Josephson vortex will form at and penetrate from the middle of the edge ( $s$. dots in Fig. 5c) rather than from the corners [14] as confirmed by magneto-optical [15] and Hallprobe experiments [16].

(ii) Due to the rectangular cross-section there is no inclination of the surface of the superconductor, and, thus, no force from vortex tension. In the absence of interaction with other flux lines, the only remaining force on the vortex is the Lorentz force generated by the Meissner screening current, $J_{M}$, flowing over the entire sample surface. In the high- $\kappa$ approximation the field drops roughly exponentially with the distance, $z$, from the edge of the superconductor, i.e. $H(z)=\mathrm{H}_{0} \mathrm{~K}_{0}(z / \lambda) \approx \mathrm{H}_{0} \exp (-z / \lambda)$. The resulting screening current shows a sharp maximum at the edge of the superconductor followed by a decrease according to $\mathrm{J}_{M}(z) \approx-\mathrm{H}_{0} / \lambda \exp (-z / \lambda)$. 
(iii) Furthermore, the magnetic induction in the junction can be estimated to be $B_{\text {eff }}=B_{0} /(1-D)$ with the demagnetization $D \approx 1-t / w$. For the $S E J$ with $w=0.7 \mu \mathrm{m}$ this yields an effective field of $B_{\text {eff }} \sim 4.4 B_{0}$ $\sim 380 \mathrm{G}$ at the field of the jumps, which exceeds the critical field $B_{c 1}$ of the superconductor, i.e. flux penetration into the electrodes is possible at $B_{0}$.

(iv) At $B= \pm \Phi_{d} /\left(w^{2} / 1.84\right.$ ) (i.e. $\sim \pm 86 \mathrm{G}$ for $\left.w=0.7 \mu \mathrm{m}\right)$ the vortex is in an unstable position at the junction and any irregularity or additional flux will force the flux line to jump into one the electrodes. As a consequence of the change in position of the flux line, the field at the junction is reduced to approximately zero as observed in the experiment. Reversing the direction of the field sweep will lead to the formation of an antivortex which will annihilate the trapped vortex in the electrode. Wider SEJ's usually do not show such pronounced jumps, since a field-free starting situation is more difficult to obtain and, thus, trapped flux will hinder the motion of flux quanta into the center of the electrode. This is also seen in the case of small SEJ's for further increase of the applied magnetic induction [10].

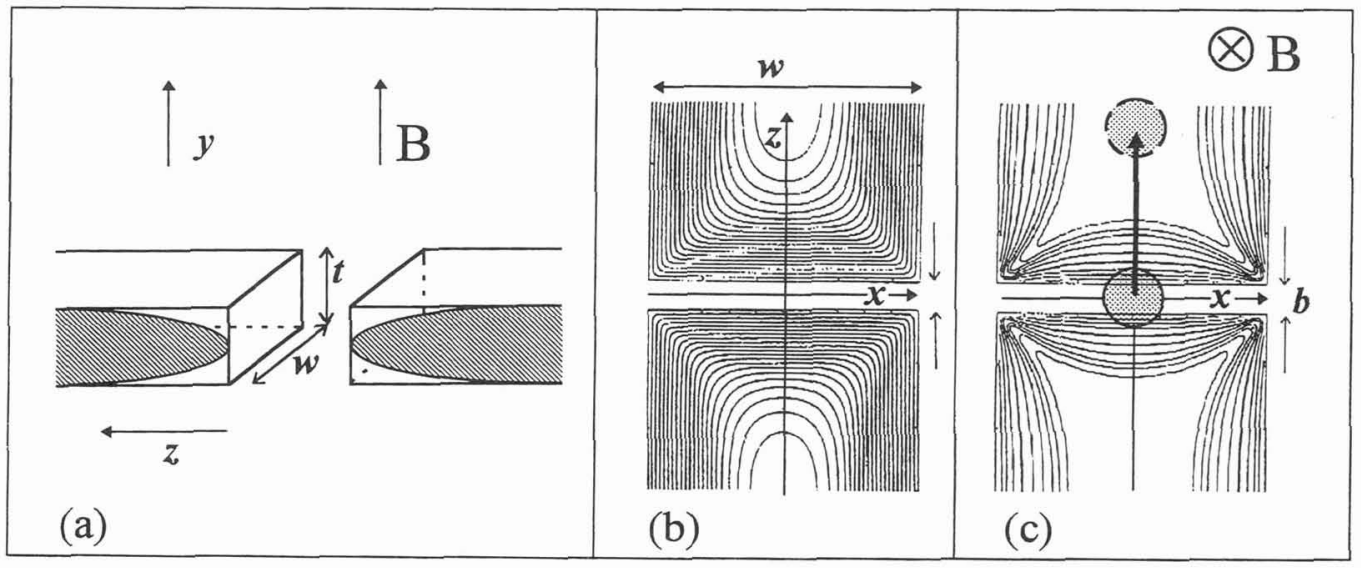

Fig. 5: (a) Sketch of rectangular and elliptical profile of the electrodes normal to the film surface, (b) topview of the current distribution and (c) magnetic field distribution in the electrodes analogously to a single crystal in Ref. [14]. The dots indicate a penetrating single Josephson vortex.

\section{SUMMARY AND OUTLOOK}

Small step-edge junctions with widths down to $w=0.5 \mu \mathrm{m}$ have successfully been fabricated in sputtered YBCO-films. Their current-voltage characteristics shows RSJ-like behavior and Shapiro steps under microwave irradiation for ratios of film thickness to step height of $t / h \approx 1 / 2$. The periodic dependence of the critical current upon the magnetic field $I_{c}(B)$ resembles a Fraunhofer-pattern. The period of the variation $\Delta \mathbf{B}_{0}$ follows a $1 / \mathrm{w}^{2}$-dependence in agreement with the theoretical prediction for planar thin $J \mathbf{J}$, i.e. $\Delta \mathbf{B}_{0}=$ $1.84 \phi_{0} / w^{2}$. SEJ's with widths of $0.7 \mu \mathrm{m}$ possess a large magnetic field stability with $\Delta \mathrm{B}_{0} \approx 70-125 \mathrm{G}$. Hysteretical behavior and reversible voltage jumps are observed at magnetic fields of $B= \pm 1.84 \Phi_{0} / \mathrm{w}^{2}$ in the Fraunhofer pattern of the small SEJ. They can be explained by flux penetration of single vortices into the superconducting electrodes.

The developed preparation technique for 'field-stable' Jo'sephson junctions will be used for the simultaneous production of a large number of magnetically stable rf-SQUIDs. The selected substrates are $\mathrm{LaAlO}_{3}$ and sapphire (r-cut) with $\mathrm{CeO}_{2}$ buffer layer. The latter is a promising material due to its high crystalline perfection, mechanical strength and low dielectric permittivity. 


\section{Acknowledgments}

The authors would like to thank A.I. Braginski for valuable comments and J. Schneider and T. Königs for technical support. Research was supported by German BMBF.

\section{References}

[1] R. W. Simon, in R. D. McConnell and Noufi (eds), Science and Technology of Thin Film Superconductors 2, Plenum Press, New York, p. 549 (1990)

[2] K. K. Likharev, Soviet Physics JETP 34, 906 (1972)

[3] P. Rosenthal, M. R Beasley, K. Char, M. S. Colclough and G. Zaharchuk, Appl. Phys. Lett. 59, 3482 (1991)

[4] R. G. Humphreys and J. A. Edwards, Physica C 210, 42 (1993)

[5] E. H. Brandt, Phys. Rev. Lett. 71, 2821 (1993)

[6] M. V. Indenbom, H. Kronmüller, T. W. LI, P. H. Kes, A. A. Menovsky, Physica C 222, 203 (1994)

[7] K. Herrmann, G. Kunkel, M. Siegel, J. Schubert, W. Zander, A. I. Braginski, C. L. Jia, B. Kabius and K. Urban J. Appl. Phys. 78, 1131 (1995)

[8] S. Beuven, M. Darula, J. Schubert, W. Zander, M. Siegel and P. Seidel, IEEE Trans. Appl. Supercond. 5, 3289 (1995)

[9] J. Schneider, H. Kohlstedt and R. Wördenweber, Appl. Phys. Lett. 63, 2426 (1993)

[10] M.Vaupel, Diploma-thesis, RWTH Aachen (1995)

[11] D. Dimos, P. Chaudhari and J. Mannhart, Phys. Rev. B 41, 4038 (1990)

[12] J. E. Evetts and B. A. Glowacki, Cryogenics 28, 641 (1988)

[13] E.H. Brandt, Phys. Rev. B (in print) 1.12.95; E.H. Brandt, Phys. Rev. Lett. 74, 3025 (1995)

[14] P. Brüll, D. Kirchgässner and P. Leiderer, Physica C 182, 339 (1991)

[15] T. Schuster et al, Phys. Rev. Lett. 73, 1424 (1994); Phys. Rev. B 50, 16684 (1994)

[16] E. Zeldov et al., Phys. Rev. Lett. 73, 1428 (1994) 